\title{
LITHIUM ABUNDANCE, DIFFUSION AND TURBULENCE
}

\author{
G. MICHAUD AND G. BEAUDET \\ Département de physique, Université de Montréal, Montréal, \\ CANADA H3C $3 J 7$
}

\section{The $\mathrm{Li}$ abundance in $\mathrm{A}$ and $\mathbf{F}$ stars}

Richer \& Michaud (1993) calculated a series of envelopes fully coupled to non-rotating, constant mass, stellar evolution models of hydrogen burning stars with masses in the range of 1.2 to $2.2 M_{\odot}$, typical of $\mathrm{A}$ and $\mathrm{F}$ main sequence stars. They included He settling. The location of the theoretically predicted gap of the Hyades agrees quite well with the observed one, a result obtained without the introduction of any free parameter. At temperatures above the gap, while the observed lithium abundances are within a factor of 2-3 of normal values, the theoretical calculated curve drops to very low values. Diffusion velocities being fairly small, any other physical process with larger or similar velocities can reduce the effect of diffusion and produce the observed results. Mass loss is one such process. Another difficulty with the present theory is the width of the gap. Observations show that the observed gap is wider than the calculated one in the Hyades. This also suggests that other physical processes play an important role.

\section{Radiative accelerations of Fe and CNO}

The diffusion of elements like helium, $\mathrm{CNO}$ and Fe, affect the stellar structure mainly through the opacity. The task of introducing helium diffusion was made easier by the existence of opacity tables with different abundances of helium. The heavy elements are normally coupled together in a variable $Z$ and only the value of $Z$ varies. If one or many elements in the group are allowed to vary independently of all the others, new opacities must be calculated. In the diffusion theory considered here, the effect of gravity is easy to calculate, but the momentum transfer of the radiation field is very complex: one needs to know precise radiative accelerations, and those depend on detailed atomic data that only recently have become available. 
Gonzalez, LeBlanc, Artru \& Michaud (1994) reviewed physical processes to be included in calculations of radiative accelerations $\left(g_{\mathrm{rad}}\right)$ and LeBlanc \& Michaud (1994) have applied these improvements in the calculation of $g_{\text {rad }}$ for iron. They found that iron is supported over a certain temperature range for $T_{\text {eff }}$ typical of A and F stars. Overabundances by factors of order 2-4 can be supported. Calculations done in the same conditions with CNO by Gonzalez et al. (1994) indicate the same type of behaviour, although at different temperatures. In both cases, it may affect stellar structure and calculations of evolutionary stellar models with diffusion should be made. For every element that diffuses, one needs about $50 \mathrm{Mb}$ of memory so there is a limit to the number of elements that can be done even with present day computers. We plan to do He, C, N, O and Fe simultaneously.

\section{Turbulent diffusion in stratified media}

The solar surface abundances for $\mathrm{Li}$ and $\mathrm{Be}$ are affected by turbulent transport between the convective zone and the location where they burn. Proffitt \& Michaud (1991) calculated solar models with turbulent diffusion that gave them upper limit values for $D_{T}$, corresponding to a Reynolds number of about 20. This is much smaller than the expected value and it challenges our understanding of the particle transport process. Turbulence is an advective process and, in the presence of a vertical concentration gradient, it can carry a net flux vertically only if there are inhomogeneities in the horizontal plane, correlated with the turbulent velocities. These may be wiped out by large horizontal motions. Vincent, Meneguzzi \& Michaud (1994) included this process in a simulation including atomic diffusion and turbulent motions in the presence of gravity. Assuming a horizontal layer of particles concentrated around the middle plane, they calculated the advection by the chosen spectrum of turbulent motions. The calculated $D_{T}$ decreased below its isotropic value with increasing anisotropy $A$, and was inversely proportional to $A$ for large $A$. So, if horizontal turbulence is much stronger than vertical turbulence in the Sun, turbulent particle transport will be inefficient in the vertical direction and this could explain why lithium was not depleted by a larger factor even if there is a relatively large amount of vertical turbulence.

\section{References}

Gonzalez, J.F., LeBlanc, F., Artru, M.-C. and Michaud, G. (1994), A \& A in press

LeBlanc, F. and Michaud, G. (1993), Astrophys. J., 408, p. 251

Proffitt, C. R. and Michaud, G. (1991), Astrophys. J., 371, p. 584

Richer, J. and Michaud, G. (1993), Astrophys. J., 416, p. 312

Vincent, A., Meneguzzi, M. and Michaud, G. (1994), submitted for publication 